\title{
Reviewer Thank You
}

\author{
Leslie R. Dye
}

Published online: 7 April 2010

(C) American College of Medical Toxicology 2010

The Editorial staff of The Journal of Medical Toxicology appreciates all of those who make the success of the journal possible. A medical journal cannot exist without the tireless, and often thankless, work of its reviewers. The list ONLY includes those reviewers registered on our web-based program, Editorial Manager, so many who have reviewed may not be on the list. To those reviewers omitted, we apologize, but also thank you very much.

Cynthia Aaron

Mohamed S. Abdel-Rahman

Redouane Abouqal

Terry Adirim

Ritesh Agarwal

Steven E. Aks

Walter Aquiles Alarcon

Timothy Albertson,

D. Adam Algren

Thomas Arnold

Gavin Arteel

Kavita Babu

Murl Bailey

Cornelia Baines

Lydia Baltarowich

Theodore Bania

Donald Barceloux

Carola Bardage

Dana Boyd Barr

Fermin Barrueto

J. Dave Barry

D. Nicholas Bateman

Stuart Batterman

Frederic Baud

\section{Martin Belson}

John G. Benitez

Phillippe J. Berny

Michael C. Beuhler

Steven B. Bird

Dennis Blodgett

G. Randall Bond

Stephen Borron

Stephen W. Borron

Mehmet Bosnak

George Bosse

Edward W. Boyer

Leslie Boyer

Eva Boyez

Gokcen Bozdag

Jeffrey Brent

Daniel E. Brooks

David Eric Brush

Sean M. Bryant

Alan Buchwald

Nick Buckley

Keith Burkhart

Howard Burns

Michael Burns
Sean Bush

Diane P. Calello

Lee Cantrell

Martin Caravati

Shaun D. Carstairs

Marcel Casavant

Edward Cetaruk

Arthur S. Chang

Nathan P. Charlton

Lu Chensheng

Roger Chou

Richard James Church

Peter Chyka

John Cienki

Cathleen Clancy

Rick Clark

Aaron Collins

James Courtney

Robert D. Cox

Barbara Insley Crouch

Kirk Lawrence Cumpston

Steven Curry

John Curtis

John Ashton Curtis

L. R. Dye $(\bowtie)$

Journal of Medical Toxicology,

10645 N. Tatum Blvd., Suite 200-111,

Phoenix, AZ 85028, USA

e-mail: lesliedye@earthlink.net 
Paul Dargan

Richard C. Dart

Cedric Daubin

Andrew Dawson

Arthur de Meijer

HJ De Silva

Kaathleen Delaney

Joao Delgado

Francis DeRoos

Christopher R. DeWitt

James Henry Diaz

Jose Diaz-Alcala

Ricardo Dinis-Oliveira

Robert Paul Dowsett

Anna Rouse Dulaney

Eric Dunayer

Francois Durand

Michael Eddleston

David Eldridge

Andrew Erdman

Michele Burns Ewald

Mariya Faheem Farooqi

Miguel Fernandez

Jeffrey Fine

Mathias B. Forrester

David Gaist

Martin Ganzert

Concetta Gardi

Ann-Jeannette Geib

Richard Geller

Robert Jerome Geller

Beth Ginsburg

Barry Gold

Daniel A. Goldstein

Christopher Gordon

Ronald Gots

Andis Graudins

Howard Andrew Greller

David Gummin

Leon Gussow

Jason Hack

In-Hei Hahn

Alan H. Hall

Christine Haller

Fred Harchelroad

Martyn Harvey

Bryan Hayes

Kennon Heard

Robert Hendrickson

Fred Henretig

James Hillman

Michael Hodgman

Robert J. Hoffman
Robert Steven Hoffman

Joel Holger

Michael Holland

Christopher Holstege

William Holubek

Mary Ann Howland

Oliver Hung

Katie Hurlbut

Danyal Ibrahim

Geoffrey Isbister

Kelly Johnson-Arbor

Heath Jolliff

James Hughes Jones

Jerry Jones

Prashant Joshi

Bryan Judge

Louise Kao

Kenneth Katz

Ziad Kazzi

Anne-Maree Kelly

Catherine Kelly

William II Kerns

D. Chris Keyes

Eun-Joo Kim

Paul G. King

Mark Kirk

Ron Kirschner

Craig Sims Kitchens

Kurt C. Kleinschmidt

OR Koch

AleÅ; Kogoj

Michael Kosnett

Mark Kostic

Edward Krenzelok

Allyson Kreshak

Edwin Kuffner

Grant Lackey

Melisa Lai

Eric J. Lavonas

Jerrold B. Leikin

Vivian Leung

Michael Levine

Howard Levitin

Philippe Lheureux

Erica Liebelt

Ja-Liang Lin

Toby Litovitz

Heather Long

Sara Monica Long

J. D. Lorenc

Jeffrey Love

Frank Lovecchio

Debbie 1 LoVerde
Jennifer Lowry

Po-Yung Lu

Andres Moran Lugo

Binh Ly

Gerald Maloney

Alex F. Manini

Kathy Marquardt

Zarko Martinovic

Michael Matteucci

Maryann Elizabeth Mazer

Fred McDonald

Charles Alan McKay

Nathanael McKeown

Bruno Megarbane

William Joel Meggs

M. John Mendelsohn

Melissa A. Miller

Michael Miller

Kirk Mills

Alicia Minns

David L. Morgan

Michelle Mostrom

Rita Mrvos

Michael Mullins

David Munter

Mike Murphy

Mark B. Mycyk

Jamie Nelsen

Lewis Nelson

Patrice Nodet

Sean Nordt

Semir Nouira

Aryn O'Connor

Gerald Francis O’Malley

Jennifer Oakes

Fred Oehme

Kent Olson

Kai Osterberg

Kevin Osterhoudt

John Osterloh

Mary Palmer

Robert Palmer

Manish Patel

Julie Pavlin

Tamas Peredy

Jeanmarie Perrone

Scott Phillips

Jessica Pierog

Steven Pike

Anthony F. Pizon

R. Ponampalam

Robert Poppenga

Laura Elizabeth Power 
Laurie Prescott

Roy Purssel

Lawrence Quang

Davud Ray

Michael Reed

Arne Reimers

Joseph Rella

William Richardson

Brad Riley

Raymond Roberge

William O. Robertson

Renee Robinson

Gary Rockwood

M.G. Roma

Rutherfoord Rose

Patricia Rosen

Jonathan Rosenson

Annalisa Rubino

Anne-Michelle Ruha

Wilson Rumbeiha

Daniel Rusyniak

Steven Salhanick

Matthew Salzman

Anthony Scalzo

Tammi Schaeffer

Elizabeth Scharman

Jay Schauben

Joshua G. Schier

James Schmidt

Aaron Schneir

Heather Schumann

David Schwartz
Michael Schwartz

Donna Seger

Gilad Segev

Robert Seidner

Steven Seifert

Steven A. Seifert

Genna Sellers

Shahin Shadnia

Beth Shortridge

Marco L.A. Sivilotti

Carl G. Skinner

Susan Smolinske

Curtis P. Snook

Henry Alfred Spiller

Andrew Stolbach

Christine M. Stork

Mark Su

Jeffrey Suchard

Daniel Sudakin

Adeline Su-Yin Ngo

David Tanen

Asim F. Tarabar

Daniel Thau Teitelbaum

Brigham Temple

Wayne Temple

Milton Tenenbein

Jerry Thomas

Trevonne Thompson

Josef G. Thundiyil

John Tilelli

Heather Tiller

Karine Titier
Anthony Tomassoni

Rebecca Tominack

Theodore Tong

Stephen Traub

Lauren Trilli

Geoffrey T. Tucker

Susi Vassallo

Larissa I. Velez

Rais Vohra

Javier Waksman

Frank Walter

Richard Wang

William Stephen Waring

David John Watts

Paul M. Wax

Patrick L. West

Kenneth S. Whitlow

Ian Whyte

Ian Macgregor Whyte

Timothy Wiegand

Brandon Wills

Chris Winder

Mary Wittler

Amy Wolkin

David Michael Wood

Alan Woolf

Robert Wright

Mark Yarema

Eduard Zaloshnja

Christina Zhang

Deborah Zvosec 